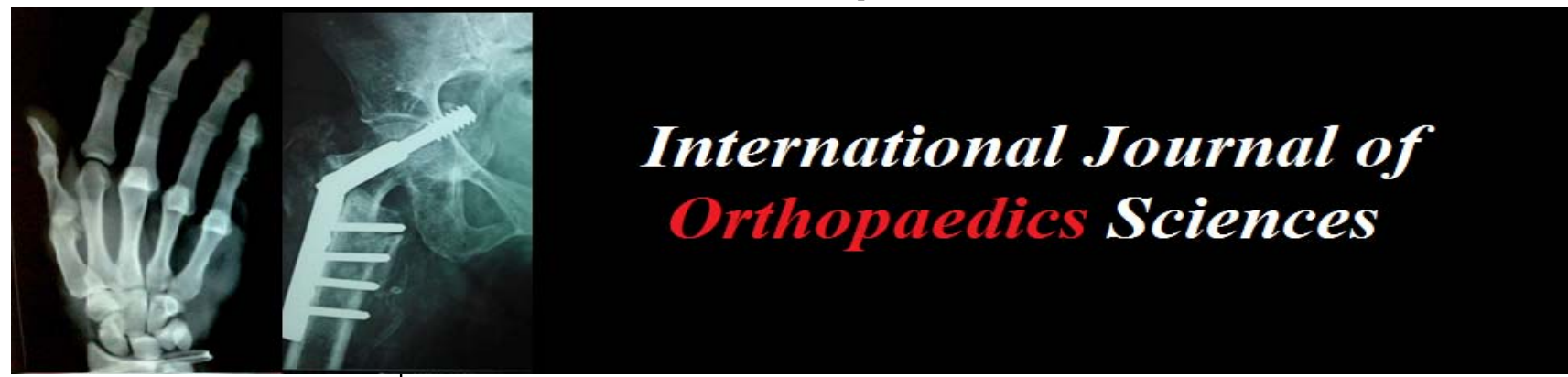

ISSN: $2395-1958$

IJOS 2017; 3(1): 75-77

(C) 2017 IJOS

www.orthopaper.com

Received: 12-11-2016

Accepted: 13-12-2016

Kumar Prashant

Medical Officer, Trauma Centre,

Banaras Hindu University,

Varanasi, U.P, India

Tulsi Das Bhattacharyya

Professor and Head, Department

of Orthopaedics, Gauhati

Medical College \& Hospital,

Guwahati, Assam, India

Nayana Bhuyan

General Physician, Varanasi,

U.P, India
Correspondence

Kumar Prashant

Medical Officer, Trauma Centre,

Banaras Hindu University,

Varanasi, U.P. India

\section{Radiodiagnostic dilemmas of telangiectatic osteosarcoma: A case report}

\author{
Kumar Prashant, Tulsi Das Bhattacharyya and Nayana Bhuyan
}

DOI: http://dx.doi.org/10.22271/ortho.2017.v3.i1b.12

Abstract

The telangiectatic osteosarcoma (TGS), an extremely lytic and hemorrhagic variant of the osteosarcoma, presents in the same age group and location as a classical osteosarcoma but has a radiographic appearance almost identical to that of an aggressive aneurismal bone cyst, making diagnosis more complex. Here we are reporting a rare case of biopsy proven telangiectatic osteosarcoma in distal tibia of a 12 years old boy, who was misdiagnosed clinically as a case of aneurismal bone cyst.

Keywords: Telangiectatic osteosarcoma, radiodiagnosis, magnetic resonance imaging

\section{Introduction}

Telangiectatic osteosarcoma (TOS) is a rare malignant variant of osteosarcoma, comprising 2 to $12 \%$ of all osteosarcomas cases ${ }^{[1-4]}$. Though it was first described by Paget ${ }^{[5]}$ in 1854 , it was Ewing ${ }^{[6]}$, who considered the tumor as a distinct histologic varient of osteosarcoma. The survival rate of TOS patients is similar to that of other osteosarcoma subtype patients ${ }^{[1-3]}$. Age, gender, tumour size and location are not predictors of non-metastatic TOS ${ }^{[4,7]}$. Because of its resemblance to aneurismal bone cyst, giant cell tumour, angiosarcoma and Ewing sarcoma at the time of radiographic evaluation, and secondly due to lack of proper clinical, radiological and pathological elucidation, its become difficult to timely diagnose and treat this rare malignant bone tumour. Here we are reporting a rare case of telangiectatic osteosarcoma in distal tibia of a 12 years old boy which was misdiagnosed as a case of aneurismal bone cyst in first instance.

\section{Case report}

A previously healthy 12-year old boy presented in our outpatient department with a 6-month history of continuous, mild initially but progressively increased in severity, left leg pain. The physical examination revealed $8 \times 5 \mathrm{cms}$ soft to firm, mild tender swelling in distal part of left leg. There was also a $2 \times 3 \mathrm{cms}$ healed ulcer on mid anterior aspect of the mass (Fig. 1). A complete blood count and basic metabolic panel were normal other than mild anaemia and mild leukocytosis. Anteroposterior and lateral radiographs of the left leg revealed an expansive septate lytic lesion in the distal tibial at metaphyseo-diaphyseal region with a narrow zone of transition. There was destruction and thinning of cortex, leaving only distal articular portion of tibia (Fig. 2). There was soft tissue spread as a result of cortical breach. Periosteal reaction was well evident. Distal fibula showed signs of mass effect. Selected T1 and T2 weighted coronal and axial MR images of the distal left tibia revealed a mass located eccentrically at the distal metaphyseo-diaphyseal region of tibia. The lesion was mixed heterogenous with fluid filled spaces (hyperintense in T2). The wide extent of diaphyseal involvement, soft tissue extent and periosteal reaction was more evident in MRI (Fig. 3). Gross and histopathological examination of mass after wide excision, confirmed it to be a telangiectatic variant of osteosarcoma (Fig. 4).

\section{Discussion}

In a series of bone tumors at the Mayo Clinic, telangiectatic osteosarcoma constituted $3.5 \%$ of all osteosarcomas and $0.7 \%$ of all malignant bone tumors ${ }^{[8]}$. 
The tumor is seen slightly more often in males (3:2 male: female ratio) and occurs most frequently in the second decade of life ${ }^{[7]}$. Similar to conventional osteosarcoma, the majority of lesions are metaphyseal and located around the knee (distal femur, proximal tibia). The pelvis, scapula, ribs, and skull are less common locations for telangiectatic osteosarcoma ${ }^{[7-9]}$. There are reports of telangiectatic osteosarcoma as a secondary tumor in Paget's disease and parosteal osteosarcoma ${ }^{[9-11]}$.

Conventional radiographs of patients with telangiectatic osteosarcoma typically reveal a lucent lesion with a wide zone of transition. Another feature described in the literature is oblique parallel striations seen in the shaft probably due to hypertrophy of veins of cortex, which cross the cortical bone in an oblique direction ${ }^{[12,13]}$. Metaphyseal lesions do extend into epiphysis. Aggressive radiographic features, such as periosteal reaction, cortical destruction, pathologic fracture, and soft-tissue mass are also common findings ${ }^{[14]}$. Consistent with the cystic nature of the tumor, the computed tomographic (CT) attenuation of the central portion of the lesion can be lower than that of soft tissue, and bone scintigraphy often shows increased radiotracer uptake peripherally in the tumor with central photopenia (known as the donut sign) ${ }^{[9]}$. Fluidfluid levels are often seen on CT and magnetic resonance imaging (MRI), but these are not entirely specific for telangiectatic osteosarcoma. Hemorrhage is frequently observed in association with the tumor as high signal intensity on T1- and T2-weighted images ${ }^{[15]}$.

Differential considerations for telangiectatic osteosarcoma during the radiographic evaluation include aneurysmal bone cyst, giant cell tumor, Ewing's sarcoma, angiosarcoma, and solitary bone cyst. Murphey et al. ${ }^{[9]}$ have reported that when subtle osteoid formation is seen in the peripheral portion of the tumor on CT or when enhancement with intravenous contrast is seen on CT or MRI in the same region, telangiectatic osteosarcoma can be differentiated from aneurismal bone cyst, which is the lesion most often confused with telangiectatic osteosarcoma.

Telangiectatic osteosarcoma is a rare malignant subtype of osteosarcoma. Combination of clinical characteristic, radiological and pathological examination can help improve the clinical diagnosis rate. The location, prognosis, and treatment of this unusual lesion mimic that of conventional osteosarcoma.

Fluid-fluid levels and areas of hemorrhagic necrosis are typically seen on MRI along with aggressive radiographic features, such as periosteal reaction, wide zone of transition, cortical destruction, and soft-tissue mass. Because of preoperative pathologic fracture and fast progression, along with diagnostic dilemma telangiectatic osteosarcoma has a higher amputation rate than other subtypes of osteosarcoma

\section{Figure Legends}

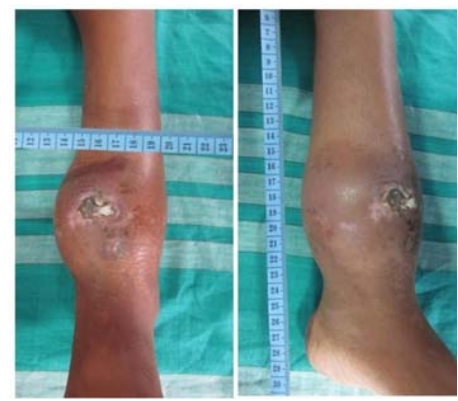

Fig 1: Clinical dimensions of swelling above ankle with skin involvement in 12 years old boy

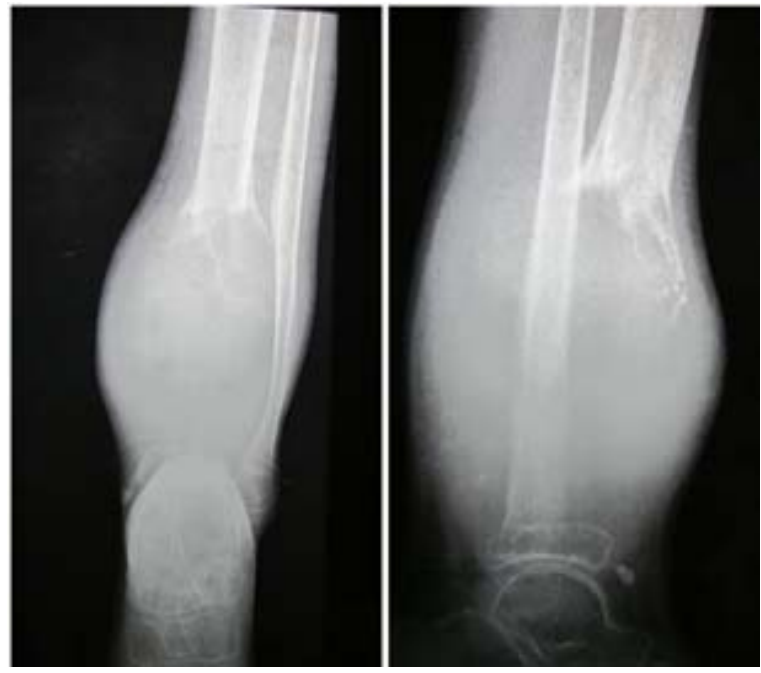

Fig. 2: Radiograph of the left both bone leg with ankle AP and Lateral view showing expansive lytic lesion in metaphysio-diaphyseal region, periosteal reaction and cortical breach with soft tissue involvement
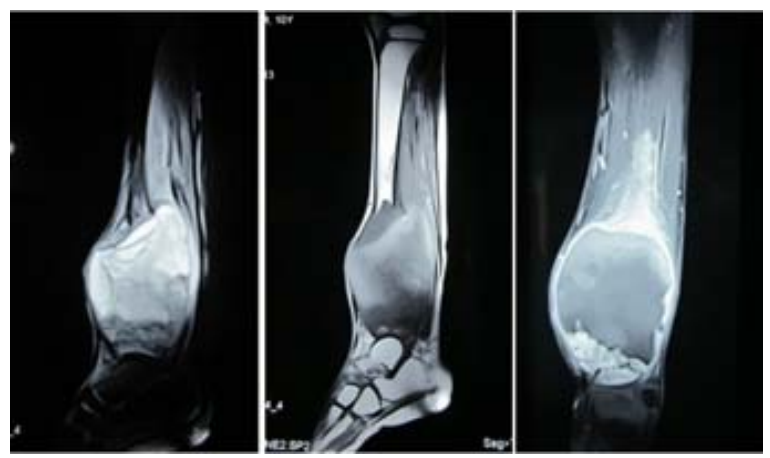

Fig 3: T1WI and T2WI MRI axial section showing mixed heterogenous with fluid filled spaces cystic composition of lesion, Hyperintensity caused by cystic lesion is better appreciated on T2WI.
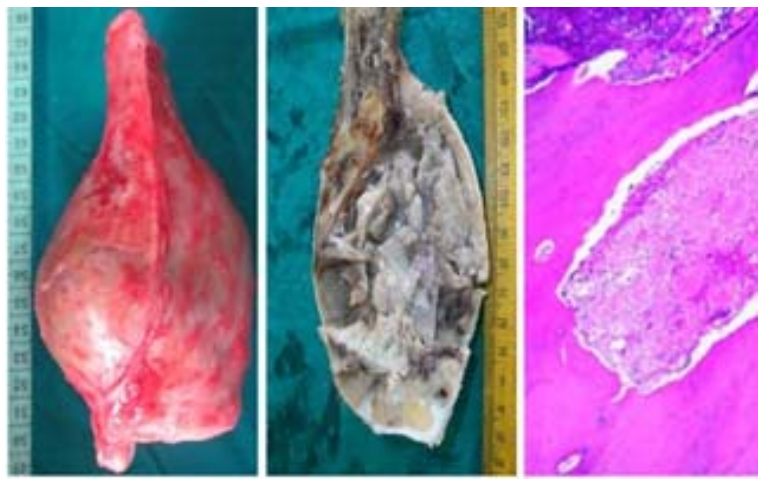

Fig 4: Gross appearance of the tumour, cut section and histopathology

\section{Funding: None}

Consent: Written informed consent from father of the child was taken for publication of this case report and accompanying images.

Competing interests: the authors declare that they have no competing interests.

Human and Animal Rights: Ethical standards followed the responsible committee on human experimentation (institutional and national) and with the Helsinki declaration of 1975 , as revised in 2000(5). 


\section{References}

1. Farr GH, Huvos AG, Marcove RC, Higinbotham NL, Foote FW Jr. Telangiectatic osteogenic sarcoma. A review of twentyeight cases. Cancer. 1974; 34:1150-8.

2. Matsuno T, Unni KK, McLeod RA, Dahlin DC. Telangiectatic osteogenic sarcoma. Cancer. 1976; 38:2538-47.

3. Weiss A, Khoury JD, Hoffer FA, Wu J, Billups CA, Heck RK et al. Telangiectatic osteosarcoma: the St. Jude Children's Research Hospital's experience. Cancer. 2007; 109:1627-37.

4. Rosen G, Huvos AG, Marcove R, Nirenberg A. Telangiectatic osteogenic sarcoma. Improved survival with combination chemotherapy. Clin Orthop Relat Res. 1986; 207:164-73.

5. Paget J. Lectures on Surgical Pathology. Philadelphia, PA: Lindsay \& Blackiston. 1854, 486.

6. Ewing J. A review and classification of bone sarcomas. Arch Surg. 1922; 4:483-533.

7. Huvos AG, Rosen G, Bretsky SS, Butler A. Telangiectatic osteogenic sarcoma: a clinicopathologic study of 124 patients. Cancer. 1982; 49:1679-89.

8. Unni KK. Dahlin's Bone Tumors: General Aspects and Data on 11,087 cases. Philadelphia, PA: LippincottRaven. 1996.

9. Murphey M, Robbin M, McRae G et al. The many faces of osteosarcoma. RadioGraphics. 1997; 17:1205-1231.

10. Merino S, Arrazola J, Saiz A et al. Post-Paget telangiectatic osteosarcoma of the skull. Skeletal Radiol. 1999; 28:470-472.

11. Wines A, Bonar F, Lam $\mathrm{P}$ et al. Telangiectatic dedifferentiation of a parosteal osteosarcoma. Skeletal Radiol. 2000; 29:597-600.

12. Johan L Bloem, Herman M Kroon. Osseous lesions: Telangiectatic osteosarcoma RCNA 1993; 31(2).

13. vanel D, Tcheng S, Contesso G, Zafrani B, Kalifa C, Dubousset $\mathrm{J}$ et al. The radiological appearance of Telangiectatic osteosarcoma Study of 14 cases Skeletal Radiology. 1987; 16:196-200.

14. Gomes H, Menanteau B, Gaillard D, Behar C. Telangiectatic Osteosarcoma Pediatr Radiol. 1986; 16:140-143.

15. Tsai JC, Dalinka MK, Fallon MD et al. Fluid-fluid level: A nonspecific finding in tumors of bone and soft tissue. Radiology. 1990; 175:779-782. 